\title{
DDX3X Gene
}

National Cancer Institute

\section{Source}

National Cancer Institute. DDX3X Gene. NCI Thesaurus. Code C101565.

This gene is involved in RNA binding. 